\title{
Synthesis of an Extended Kalman Filter for Cable-Driven Parallel Robots
}

\author{
Giovanni Boschetti ${ }^{1}$, Francisco González ${ }^{2}$, Giulio Piva ${ }^{1}$, Dario Richiedei ${ }^{1}$, Borja R. Frade ${ }^{2}$, \\ Alberto Trevisani ${ }^{1}$ \\ ${ }^{1}$ Department of Management and Engineering \\ University of Padua \\ Stradella S. Nicola 3, 36100, \\ Vicenza, Italy \\ [giovanni.boschetti, dario.richiedei, alberto.trevi- \\ sani]@unipd.it, \\ giulio.piva@phd.unipd.it \\ ${ }^{2}$ Laboratorio de Ingeniería \\ Mecánica \\ University of A Coruña \\ Rúa Mendizábal s/n, 15403, \\ Ferrol, Spain \\ [f.gonzalez, \\ borja.rfrade]@udc.es
}

\begin{abstract}
Cable-driven parallel robots are light-weight parallel robots where cables replace rigid actuators to move an end-effector. As a consequence, they can be characterized by very large workspaces, high-dynamic handlings, ease of reconfigurability and/or low-cost architecture. Since the driving links are flexible, the state variables of the robot cannot be always directly measured, thus the development of state observers is essential. In this work a general approach to develop a nonlinear state observer based on an Extended Kalman Filter is proposed and validated numerically by referring to a cable-suspended parallel robot. The state observer is based on a system model obtained converting a set of Differential Algebraic Equations into Ordinary Differential Equations through two different methods: the penalty formulation and the Udwadia-Kalaba formulation.
\end{abstract}

Keywords: State estimation, Extended Kalman Filter, Cable-Driven Parallel Robots, Penalty Formulation, Udwadia-Kalaba.

\section{INTRODUCTION AND MOTIVATION}

Parallel robots (e.g., the Stewart-Gough platform) typically actuate the end-effector by driving rigid links, in Cable-Driven Parallel Robots (CDPRs), conversely, the end-effector is actuated by flexible cables. Parallel robots can be designed to achieve high stiffness at the end-effector and payload capacity, but the weight of the actuators and their fixed minimum and maximum lengths may limit considerably the feasible velocity and the workspace. Alternatively, parallel robots can be designed to achieve high velocity at the expenses of payload capacity, but the workspace limitations cannot be overcome (e.g., Delta robots). In CDPRs, instead, each cable is wound around a winch connected to a motor. The winch can easily provide several meters of cable not only enabling large workspaces, but also assuring minimal inertias, lightweight structures, easy reconfigurability and modularity of the system.

Despite these advantages, the current deployment of CDPRs seems hampered mainly by the fact that cables are unilateral elements: they can only exert pulling forces. If they become slack, the end-effector cannot be made follow a prescribed trajectory or exert the required wrench to perform a task: in manufacturing or heavy handling, this can obviously introduce relevant performance and safety issues since the control of the end-effector may be lost [1]. The use of CDPRs has, however, already been suggested in several different operation fields, such as heavy handling, medical rehabilitation, rescue and home assistance, industrial manufacturing, or sport shooting (see for example [2] and the references therein). In the future, a wide use of CDPRs can be foreseen thanks to their lightweight structure (which makes them energy efficient), modularity and reconfigurability (which makes them flexible and easy to transport) and finally, the potentially high dynamics and payload capacity (which makes them effective in a wide range of industrial applications). The presence of flexible elements can however introduce accuracy issues as a 
consequence of cable elasticity and sagging. Additionally, the upper and lower bounds on admissible cable tensions impose implementing complex planning and motion control strategies. These problems clearly demand developing and using accurate dynamic models for CDPRs.

There is no single classification universally recognized for CDPRs, not even the terminology adopted is unified. CDPRs topologies can however be very dissimilar, and every topology presents advantages and drawbacks that must be considered at the design stage and when developing planning and control algorithms. To try to simplify the analysis the main categories shown in Table 1 can be used to classify CDPRs.

Table 1. Classifications of CDPRs

\begin{tabular}{cccc}
\hline Workspace & Actuation & Constraints & Exit-points \\
\hline Planar & Under-actuated & Under-constrained & Fixed \\
Spatial & Fully-actuated & Fully-constrained & Reconfigurable \\
& Over-actuated & Redundant & \\
&
\end{tabular}

As for the workspace, a CDPR can obviously operate in a three-dimensional space or into a bidimensional (planar) space.

As for the actuation, if the end-effector of a CDPR is driven by $n_{a c}$ active cables, depending on the number of degrees of freedom, $n_{d o f}$, of the end-effector, the CDPR can be:

- Under-actuated if $n_{a c}<n_{d o f}$ [3]

- Fully-actuated, if $n_{a c}=n_{d o f}[4]$;

- Over-actuated, if $n_{a c}>n_{d o f}[5]$.

Another critical feature of a CDPR is the capability of cables to react to external forces acting on the end-effector. Different cases can be recognized:

- Under-constrained: there exist some directions along which cables cannot exert equilibrating reactions to external wrenches applied to the end-effector [6];

- Fully-constrained: the end-effector can maintain equilibrium against every external wrench (under the assumption of infinite maximum cable tension) [7];

- Redundant: the number of cables is greater than the minimum one making the robot fullyconstrained [8].

Finally, CDPRs can also present reconfiguration capabilities for their exit points (i.e., the points on the opposite side of the cable with respect to the points connected to the end-effector). Typically, the exit points are fixed, but more in general, they can follow the motion of, for example, drones [9], motors moving along a fixed frame [10] or even other manipulators. These robots are called Reconfigurable Cable-Driven Parallel Robots (RCDPRs) and are expected to have interesting applications also in the rescue and home assistance fields.

A critical issue in the field of cable robotics is ensuring that cable tensions are positive. Therefore, proper motion planning and control should be performed by adopting advanced techniques. In the case of feedback control, advanced control schemes often require the knowledge of the full state of the system (see e.g. [11,12]), that is in contrast usually not available. To overcome this issue, state observers can be designed and implemented [13,14].

In this paper, an Extended Kalman Filter (EKF) is adopted to estimate the state variables of a CDPR. The dynamics of a CDPR is highly nonlinear and imposes the development of nonlinear state observer, such as EKFs, which are widely used in state estimation of nonlinear systems starting from a complete model formulated through first-order Ordinary Differential Equations (ODEs). A widespread approach in the literature of CDPRs is writing the Newton-Euler equations of motion for the end-effector under the hypothesis that cables are stiff, massless and straight, and often the contribution of the motor inertial properties to the overall system dynamics is neglected, or just considered through approximations or through non-systematic approaches. On the other hand, redundant coordinates are never used in dynamic models, although this choice has several 
benefits in modelling this CDPRs (e.g. to simulate cable failures or bouncing motions), and also make easier the development of models including both the motors and the end-effector. The latter is therefore the approach investigated in this work for the design of state observers.

Since the models implemented in the state observer need to be in ODEs representation, the conversion of DAEs into ODEs must be performed. This step can be carried out taking advantage of different formulations. In this work, two well established formulations are investigated: the penalty formulation [15] and the Udwadia-Kalaba formulation [16], since both the approaches are well suited for handling multibody systems with redundant constraints [15,17], as often occurs in CDPRs.

\section{DYNAMIC MODELING OF A CABLE-DRIVEN PARALLEL ROBOT}

\subsection{Differential-Algebraic Equations (DAEs) modeling technique}

Let us collect the coordinates of a CDPR into the vector of dependent coordinates $\mathbf{q} \in \mathbb{R}^{p}$. The following set of DAEs, of index 3 , is obtained to model the system dynamics [18]:

$$
\left\{\begin{array}{c}
\mathbf{M} \ddot{\mathbf{q}}+\mathbf{J}^{T} \lambda=\mathbf{f} \\
\mathbf{\Phi}(\mathbf{q})=\mathbf{0}
\end{array}\right.
$$

where $\boldsymbol{\Phi}(\mathbf{q})$ is the set of $n$ kinematic constraint equations, $\mathbf{M} \in \mathbb{R}^{p \times p}$ is the mass matrix, $\mathbf{f} \in \mathbb{R}^{p}$ is the vector of the external forces, $\lambda \in \mathbb{R}^{n}$ is the vector of the Lagrange multiplier and $\mathbf{J} \in \mathbb{R}^{n \times p}$ is the Jacobian of the constraint equations, $\mathbf{J}=\left[\frac{\partial \mathbf{\Phi}}{\partial \mathbf{q}}\right]$.

The CSPR studied in this work, as sketched in Figure 1, is a cable-suspended robot: a three-DOF suspended end-effector (modeled as a lumped mass $m$ ) is driven by four cables winding on winches and actuated by motors (whose equivalent moments of inertia reflected to the motor shaft are $\left.J_{m, 1}, J_{m, 2}, J_{m, 3}, J_{m, 4}\right)$. The system is therefore overactuated, as often happens in CDPRs, since this configuration increases the static equilibrium workspace [19]. Vector $\mathbf{q}=\left[\begin{array}{ll}\mathbf{p}^{T} & \boldsymbol{\theta}^{T}\end{array}\right]^{T}$ includes the absolute Cartesian positions of the end-effector $\mathbf{p}=\left[\begin{array}{lll}x_{p} & y_{p} & z_{p}\end{array}\right]^{T}$ and the angular positions of the motors $\boldsymbol{\theta}=\left[\begin{array}{llll}\theta_{1} & \theta_{2} & \theta_{3} & \theta_{4}\end{array}\right]^{T}$. Under the assumption that cables are perfectly stiff and taut, and hence behave as holonomic, ideal kinematic constraints, the $i$-th constraint $(i=1, . ., 4)$ that relates the end-effector coordinates and the angular positions of the motors is $\mathbf{l}_{i}=\mathbf{p}-\mathbf{a}_{\mathbf{i}}$ (with $\mathbf{a}_{\mathbf{i}}$ the absolute position of the exit-point of cable $i$ ), where $\left\|\mathbf{I}_{\mathbf{i}}\right\|=l_{0, i}+r_{i} \theta_{i}$ is the $i$-th cable length $\left(l_{0, i}\right.$ is the $i$-th cable length corresponding to $\theta_{i}=0$ and $r_{i}$ is the radius of the $i$-th winch).

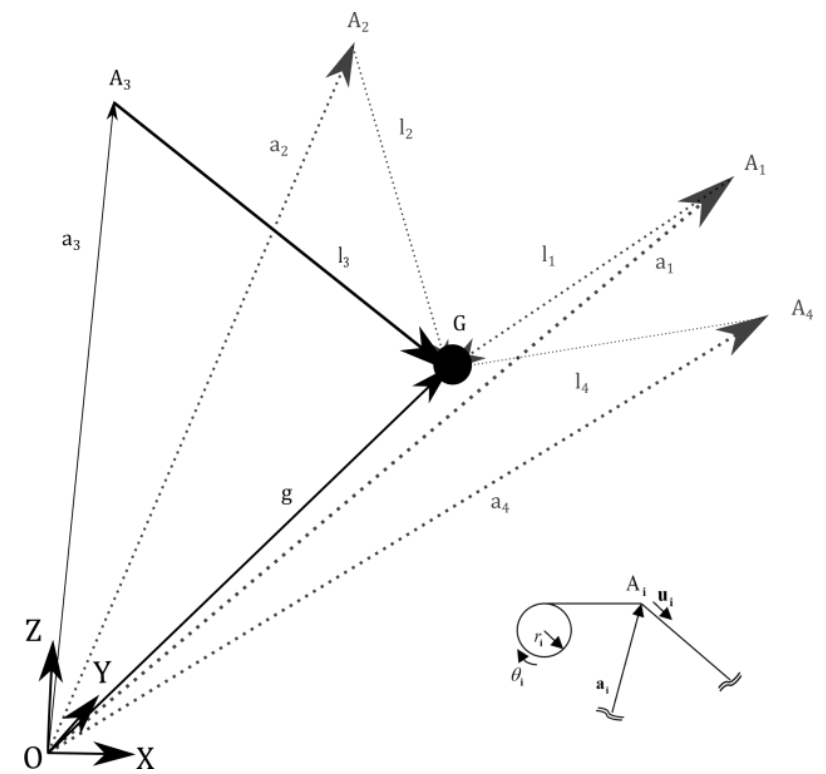

Figure 1. CSPR driven by four cables and detail of the $i$-th winch and exit point. 
The $i$-th position constraint can therefore be expressed in the following form:

$$
\Phi_{i}=\mathbf{p}^{T} \mathbf{p}-2 \mathbf{p}^{T} \mathbf{a}_{\mathbf{i}}+\mathbf{a}_{\mathbf{i}}^{T} \mathbf{a}_{\mathbf{i}}-l_{0, i}{ }^{2}-2 l_{0, i} r \theta_{i}-r^{2} \theta_{i}^{2}=0
$$

The conversion of the set of DAEs to a set of nonlinear ODEs, both for simulating the system and for designing the state observer, can be done through different approaches. Three different approaches are here considered, as briefly discussed in Sections 2.2, 2.3 and 2.4.

\subsection{DAE to ODE conversion: projection matrix}

A usual way to convert a DAE model into a minimal set of ODE is using the "projection matrix method", by means of the matrix (here denoted as $\mathbf{R} \in \mathbb{R}^{\left(p-n_{\text {dof }}\right) \times p}$ ) that relates the dependent (redundant) velocities, $\dot{\mathbf{q}}$, and the independent (minimal) ones, $\dot{\mathbf{z}}$ :

$$
\dot{\mathbf{q}}=\mathbf{R}(\mathbf{q}) \dot{\mathbf{z}}
$$

taking the time-derivative of Eq. (3), $\ddot{\mathbf{q}}=\mathbf{R}(\mathbf{q}) \ddot{\mathbf{z}}+\dot{\mathbf{R}}(\mathbf{q}, \dot{\mathbf{q}}) \dot{\mathbf{z}}$, leads to the minimal set of ODEs

$$
\mathbf{R}(\mathbf{q})^{T} \mathbf{M R}(\mathbf{q}) \ddot{\mathbf{z}}=\mathbf{R}(\mathbf{q})^{T}(\mathbf{f}-\mathbf{M} \dot{\mathbf{R}}(\mathbf{q}, \dot{\mathbf{q}}) \dot{\mathbf{z}})
$$

Eq. (4) can be expressed in the following compact form, with the obvious meaning of the symbols:

$$
\overline{\overline{\mathbf{M}}} \ddot{\mathbf{z}}=\overline{\overline{\mathbf{f}}}
$$

\subsection{DAE to ODE conversion: Penalty Formulation}

To retain all the redundant coordinates in the multibody model, a formulation often used to convert the DAEs model to an ODEs system is using the "Penalty Formulation", which allows directly simulating the time evolution of all the dependent coordinates [18]. The penalty formulation assumes that the Lagrange multipliers are proportional to the constraint violation at the configuration, velocity, and acceleration levels. In its simplest form, the following definition is assumed, by means of the three scalar tuning parameters $\alpha, \xi$ and $\omega$.

$$
\lambda=\alpha\left(\ddot{\boldsymbol{\Phi}}+2 \xi \omega \dot{\boldsymbol{\Phi}}+\omega^{2} \mathbf{\Phi}\right)
$$

The choice of their values has been carried out by following the advices proposed in the literature (see e.g. [15]); if the model is used in the design of a state observer, they can be treated as two tuning parameters. Since the constrains of the system under investigation are scleronomic, the time-derivative of the position constraints can be expressed as

$$
\begin{gathered}
\dot{\boldsymbol{\Phi}}(\mathbf{q})=\mathbf{J} \dot{\mathbf{q}} \\
\ddot{\boldsymbol{\Phi}}(\mathbf{q})=\mathbf{J} \ddot{\mathbf{q}}+\dot{\mathbf{J}} \dot{\mathbf{q}}
\end{gathered}
$$

and therefore, the following set of $p$ ODEs is obtained to model the dynamics of the CDPR:

$$
\left(\mathbf{M}+\mathbf{J}^{T} \alpha \mathbf{J}\right) \ddot{\mathbf{q}}+\mathbf{J}^{T} \alpha(\dot{\mathbf{J}}+2 \xi \omega \mathbf{J}) \dot{\mathbf{q}}+\mathbf{J}^{T} \alpha \omega^{2} \mathbf{\Phi}=\mathbf{f}
$$

again, Eq. (8) can be written in the following compact form

$$
\overline{\mathbf{M}}(\mathbf{q}) \ddot{\mathbf{q}}+\overline{\mathbf{C}}(\mathbf{q}) \dot{\mathbf{q}}+\overline{\mathbf{K}}(\mathbf{q})=\mathbf{f}
$$

\subsection{DAE to ODE conversion: Udwadia-Kalaba formulation}

A different approach to convert the DAEs into a set of ODEs retaining all the $p$ dependent coordinates is through the methods exploiting the exact evaluation of the Lagrange multipliers, most of which are related to the Gauss' principle of least constraint. The most famous of these methods is, probably, the Udwadia-Kalaba formulation [20], that holds for systems with non- 
singular mass matrix. The acceleration of the $p$ dependent coordinates of the constrained system, $\ddot{\mathbf{q}}$, is obtained as the sum of the free-body (unconstrained) accelerations $\ddot{\mathbf{q}}_{f}$,

$$
\ddot{\mathbf{q}}_{f}=\mathbf{M}^{-1} \mathbf{f}
$$

and a perturbation due to the kinematic constraints, $\ddot{\mathbf{q}}_{c}$, that can be computed as follows:

$$
\ddot{\mathbf{q}}_{c}=\mathbf{M}^{-\frac{1}{2}} \mathbf{B}^{\dagger}\left(\boldsymbol{\Gamma}-\mathbf{J} \ddot{\mathbf{q}}_{f}\right)
$$

matrix $\mathbf{B}^{\dagger}$ is the pseudoinverse of $\mathbf{B}$, with

$$
\mathbf{B}=\mathbf{J M}^{-\frac{1}{2}},
$$

while $\Gamma$ is due to the acceleration constraint equations:

$$
\boldsymbol{\Gamma}=-\frac{\partial(\mathbf{J} \dot{\mathbf{q}})}{\partial \mathbf{q}} \dot{\mathbf{q}}-2 \dot{\mathbf{J}} \dot{\mathbf{q}}-\ddot{\boldsymbol{\Phi}}
$$

since this formulation arises from a DAE system of index 1, position and speed constraints usually are not satisfied after numerical integration of the equation of motions. Therefore, the Baumgarte stabilization [21] is usually introduced, by leading to the following set of ODEs to be integrated:

$$
\ddot{\mathbf{q}}=\mathbf{M}^{-1} \mathbf{f}+\mathbf{M}^{-\frac{1}{2}} \mathbf{B}^{\dagger}\left(\boldsymbol{\Gamma}-2 \chi \dot{\boldsymbol{\Phi}}-\varphi^{2} \mathbf{\Phi}-\mathbf{J} \ddot{\mathbf{q}}_{f}\right)
$$

where $\chi$ and $\varphi$ are Baumgarte stabilization parameters that are tuned with a trial-and-error procedure. If the model is used in the design of a state observer, they could be treated as two tuning parameters.

Whenever $\mathbf{M}$ is singular, the extension of this formulation provided in [22] could be exploited.

\section{DEVELOPMENT OF THE EXTENDED KALMAN FILTER (EKF)}

Extended Kalman Filters (EKFs) are widely used as nonlinear state observers to estimate unmeasured variables in multibody systems [14]. An EKF provides optimal estimates $\hat{\mathbf{x}}(t)$ of the actual state $\mathbf{x}(t)=\left[\begin{array}{ll}\dot{\mathbf{q}}^{T} & \mathbf{q}^{T}\end{array}\right]^{T}$ of a first-order system representation, by merging the prediction of a nominal model $\dot{\mathbf{x}}(t)=\boldsymbol{f}_{c}(\mathbf{x}(t), \mathbf{u}(t)) \quad(\mathbf{u}(t)$ is the input vector), with a closed-loop correction inferred through the measurements retrieved from a proper set of sensors $\mathbf{y}(t)=g(\mathbf{x}(t), \mathbf{u}(t))$ ensuring observability. The resulting closed-loop estimation is based on a prediction-correction scheme, that in the continuous time leads to the following form

$$
\hat{\dot{\mathbf{x}}}(t)=\boldsymbol{f}_{c}(\mathbf{x}(t), \mathbf{u}(t))+\mathbf{L}(\mathbf{y}(t)-\hat{\mathbf{y}}(t))
$$

where $\mathbf{L}$ is the filter gain and $\mathbf{y}(t)-\hat{\mathbf{y}}(t)$ is the output-estimation error ( $\hat{\mathbf{y}}(t)$ is the estimated output), usually denoted as the innovation. In practice, the EKF is implemented in discrete-time and the vectors through the discretized form of the function $f_{c}$, here denoted as $f$ :

$$
\left\{\begin{array}{c}
\mathbf{x}_{k}=\boldsymbol{f}\left(\mathbf{x}_{k-1}, \mathbf{u}_{k-1}\right) \\
\mathbf{y}_{k}=\boldsymbol{g}\left(\mathbf{x}_{k}, \mathbf{u}_{k}\right)
\end{array}\right.
$$

where $k$ denotes the simulation step.

The discrete-time model $\boldsymbol{f}$ and the noisy input measurements $\mathbf{u}_{k}$ are adopted for computing the prediction (or a-priori estimation) $\hat{\mathbf{x}}_{k \mid k-1}=\boldsymbol{f}\left(\hat{\mathbf{x}}_{k-1 \mid k-1}, \mathbf{u}_{k}\right)$, that is then corrected through the output estimation error $\left(\mathbf{y}_{k}-\hat{\mathbf{y}}_{k \mid k-1}\right)$, with $\hat{\mathbf{y}}_{k \mid k-1}=\boldsymbol{g}\left(\hat{\mathbf{x}}_{k \mid k-1}, \mathbf{u}_{k}\right)$, weighed through the time-varying filter gain $\mathbf{L}_{k \mid k}$, leading to the following recursive scheme:

$$
\hat{\mathbf{x}}_{k \mid k}=\hat{\mathbf{x}}_{k \mid k-1}+\mathbf{L}_{k \mid k}\left(\mathbf{y}_{k}-\hat{\mathbf{y}}_{k \mid k-1}\right)
$$

The term $\mathbf{L}_{k \mid k}\left(\mathbf{y}_{k}-\hat{\mathbf{y}}_{k \mid k-1}\right)$ is a closed-loop correction, in the control theory sense, forcing the 
estimation to track sensor measurements by compensating for noise and model uncertainty. To compute $\mathbf{L}_{k \mid k}$ at each time step, the EFK algorithm replaces the nonlinear model with its Jacobian matrices computed about the estimated state trajectory and uses them in the propagation of the noise covariance matrices [14].

As an example, the EKF developed with the penalty formulation is based on Eq. (9), which allows expressing the accelerations in the following form:

$$
\ddot{\mathbf{q}}=\overline{\mathbf{M}}(\mathbf{q})^{-1} \mathbf{f}-\overline{\mathbf{M}}(\mathbf{q})^{-1} \overline{\mathbf{K}}(\mathbf{q})-\overline{\mathbf{M}}(\mathbf{q})^{-1} \overline{\mathbf{C}}(\mathbf{q}) \dot{\mathbf{q}}
$$

the following first-order representation of the system is obtained:

$$
\left[\begin{array}{c}
\ddot{\mathbf{q}} \\
\dot{\mathbf{q}}
\end{array}\right]=\left[\begin{array}{cc}
-\overline{\mathbf{M}}^{-1} \overline{\mathbf{C}} & \mathbf{0} \\
\mathbf{I} & \mathbf{0}
\end{array}\right]\left[\begin{array}{l}
\dot{\mathbf{q}} \\
\mathbf{q}
\end{array}\right]+\left[\begin{array}{cc}
-\overline{\mathbf{M}}^{-1} & \mathbf{0} \\
\mathbf{0} & \mathbf{0}
\end{array}\right]\left[\begin{array}{c}
\overline{\mathbf{f}} \\
\mathbf{0}
\end{array}\right]
$$

where the equivalent external forces vector $\overline{\mathbf{f}}$ is:

$$
\overline{\mathbf{f}}=\mathbf{f}-\overline{\mathbf{K}}(\mathbf{q})
$$

Several discretization schemes can be adopted, with different accuracy, stability and computational effort $[14,18]$. In this paper, with the goal of simplify the computational for boosting real time estimation and aware of the positive effect of the filter correction that can compensate for energy losses due to the numerical integration scheme, discretization is performed with a simplified method based on an approximation of the forward Euler scheme (with time-step $\mathrm{d} t$ ), as often done in control theory. The following state-dependent matrices are defined, due to the dependence of some submatrices on $\mathbf{q}$ and $\dot{\mathbf{q}}$ (that is omitted for clarity of representation):

$$
\begin{gathered}
\mathbf{A}_{d, k} \approx \mathbf{I}+\left[\begin{array}{cc}
-\overline{\mathbf{M}}^{-1} \overline{\mathbf{C}} & \mathbf{0} \\
\mathbf{I} & \mathbf{0}
\end{array}\right]_{k} \mathrm{~d} t \\
\mathbf{B}_{d, k} \approx\left[\begin{array}{cc}
-\overline{\mathbf{M}}^{-1} & \mathbf{0} \\
\mathbf{0} & \mathbf{0}
\end{array}\right]_{k} \mathrm{~d} t
\end{gathered}
$$

where $\mathbf{A}_{d, k}$ and $\mathbf{B}_{d, k}$ represent the discrete counterpart of the continuous-time matrices of Eq. (19). Then, the discrete-time model $\mathbf{x}_{k+1}=\boldsymbol{f}\left(\mathbf{x}_{k}, \mathbf{u}_{k}\right)$ of Eq. (18) is cast as follows:

$$
\left[\begin{array}{c}
\dot{\mathbf{q}} \\
\mathbf{q}
\end{array}\right]_{k}=\mathbf{A}_{d, k-1}\left[\begin{array}{c}
\dot{\mathbf{q}} \\
\mathbf{q}
\end{array}\right]_{k-1}+\mathbf{B}_{d, k-1}\left[\begin{array}{c}
\overline{\mathbf{f}} \\
\mathbf{0}
\end{array}\right]_{k-1}
$$

and in the following compact form (that apparently resembles the one of a linear system):

$$
\mathbf{x}_{k}=\mathbf{A}_{d, k-1} \mathbf{x}_{k-1}+\mathbf{B}_{d, k-1} \mathbf{u}_{k-1}
$$

with the obvious definitions of the state and input vectors:

$$
\mathbf{x}_{k}=\left[\begin{array}{c}
\dot{\mathbf{q}} \\
\mathbf{q}
\end{array}\right]_{k} \quad \mathbf{u}_{k}=\left[\begin{array}{l}
\overline{\mathbf{f}} \\
\mathbf{0}
\end{array}\right]_{k}
$$

By following the recursive scheme of the EKF, the covariance propagation is computed as:

$$
\hat{\mathbf{P}}_{k \mid k-1}=\mathbf{A}_{d, k-1} \mathbf{P}_{k-1} \mathbf{A}_{d, k-1}^{T}+\mathbf{Q}
$$

where $\mathbf{Q}$ is the covariance matrix of the of the model noise, that is in practice a tuning parameter that represents in an abstract way the amount of model uncertainty. Then, the filter gain is computed as

$$
\mathbf{L}_{k \mid k}=\hat{\mathbf{P}}_{k \mid k-1} \mathbf{H}^{T}\left(\mathbf{H} \hat{\mathbf{P}}_{k \mid k-1} \mathbf{H}^{T}+\mathbf{R}\right)^{-1}
$$

where $\mathbf{R}$ denotes the covariance matrix of measurement noise that can be treated as a tuning parameter, and $\mathrm{H}$ is the Jacobian of $\boldsymbol{g}$. Finally, the covariance propagation matrix is updated by setting 


$$
\mathbf{P}_{k}=\left(\mathbf{I}-\mathbf{L}_{k \mid k} \mathbf{H}\right) \hat{\mathbf{P}}_{k \mid k-1}
$$

\section{NUMERICAL RESULTS}

\subsection{Description of the test case}

The system under investigation is a Cable-Suspended Parallel Robot (CSPR), which is a particular CDPR where all the cables are connected to the end-effector from the top of the frame, and therefore the possibility to get positive tensions in the cables is just provided by gravity. The CSPR analysed as the test case is sketched in Figure 2: it is made by 4 motors that actuate the end-effector, modeled as a point mass $m=3[\mathrm{~kg}]$. The frame dimensions are $1.69 \times 1.775 \times 1.89$ $[\mathrm{m}](\mathrm{w} \times 1 \times \mathrm{h}$, in Figure 2). Therefore, it can be classified as a spatial, over-actuated, underconstrained CDPR with fixed exit-points. The exit points are assumed to coincide with the upper the vertices $\mathrm{A}_{i}$ of the frame $(i=1, \ldots, 4)$. The actuators have equal rotational moments of inertia $J_{m, i}=5.12 e-4\left[\mathrm{kgm}^{2}\right](i=1, . ., 4)$ (including both the motor rotor and the winch). Rigid and taut cables are assumed, as is often reasonable if low-frequency motions are considered.

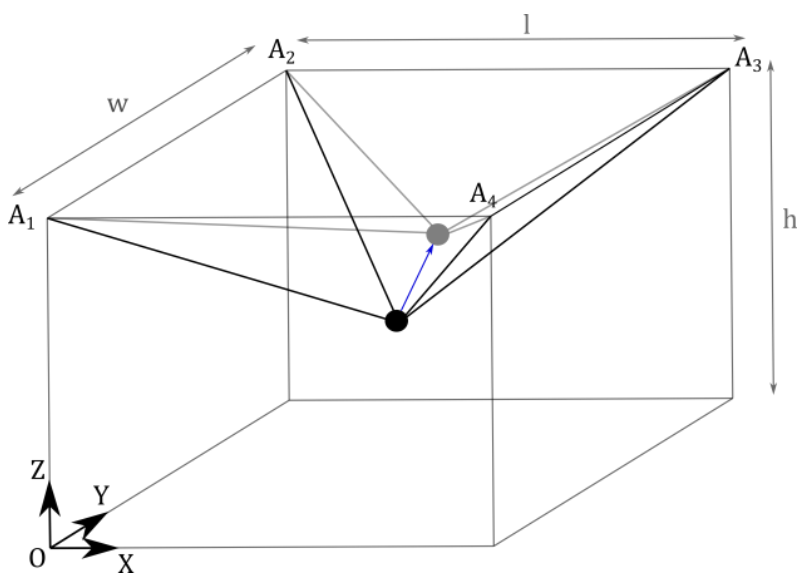

Figure 2. Basic scheme of the CSPR

The actuators are supposed to be equipped by low resolution encoder measuring $\boldsymbol{\theta}$, with just 150 pulses per revolution and operating in $4 \mathrm{x}$ resolution; such a choice ensures observability. The torques exerted by each motor is available as well, as usually supplied by commercial drivers and as required by the state observer. The simulator adopted to represent the "real system", i.e. the system that produces the "actual" values of the state vector to be estimated by the state observer, has been implemented through the projection matrix method, which uses a minimal coordinate representation, and does not require any tuning parameter in the conversion of the DAEs to ODEs, as in contrast is required by both the Penalty and the Udwadia-Kalaba formulations. The "actual" values of $\boldsymbol{\theta}$ that are fed to the state observer are corrupted by quantization noise. Additionally, some errors in the mass matrices of the models employed in the observer will be considered in Section 4.4, to assess the observer capability of getting rid of such uncertainties by merging the model and the closed loop correction. Two different EKFs are tested, by adopting the Penalty and the Udwadia-Kalaba formulations for the model-based filter prediction. In this way, the impact of different multibody formulations on the estimate accuracy is evaluated.

Besides comparing the observer outcomes with the actual state, the estimates of the end-effector position and velocities are made through the forward kinematics and the noisy measurements provided by the encoders. As for the estimation of the motor shaft speeds, it is obtained by numerical derivation and by low pass filtering through a first-order filter with a $15 \mathrm{~Hz}$ bandwidth. Increasing the bandwidth does not allow properly removing high frequency noise introduced by derivation of the encoder signal corrupted by the coarse quantization. On the other hand, such a filter creates a phase lag in the estimated speeds and therefore further reducing the bandwidth would decrease the stability margin if such estimates are used in feedback control loops. 
The simulated test consists of a rest-to-rest motion from point $P_{i}=\left\{\begin{array}{lll}0.8875 & 0.8425 & 0.9450\end{array}\right\}[\mathrm{m}]$ to point $P_{f}=\left\{\begin{array}{lll}1.0 & 1.0 & 1.5\end{array}\right\}[\mathrm{m}]$ through a linear path, as shown in Figure 2, by means of a $5^{\text {th }}$ degree polynomial law of motion.

\subsection{State observer based on penalty formulation: EKF-P}

The Cartesian coordinates of the end-effector positions $\left(x_{p}, y_{p}\right.$ and $\left.z_{p}\right)$ and velocities $\left(\dot{x}_{p}, \dot{y}_{p}\right.$ and $\dot{z}_{p}$ ) are shown from Figures from 3 through 5 . In each figure, a comparison is shown among the "real system" coordinates, the estimates of the EKF based on penalty formulation (hereafter denoted as EKF-P) and the estimations obtained through forward kinematics. The inspection of the velocity estimates reveals that the use of the EKF remarkably reduces the effect of the quantization noise on the derivatives, compared to the kinematics estimation, without introducing visible delay.

A closer look on the result can be inferred from the error plots shown in Figures 6 and 7, that are also summarized in Table 2 through the RMS (root mean square) values.
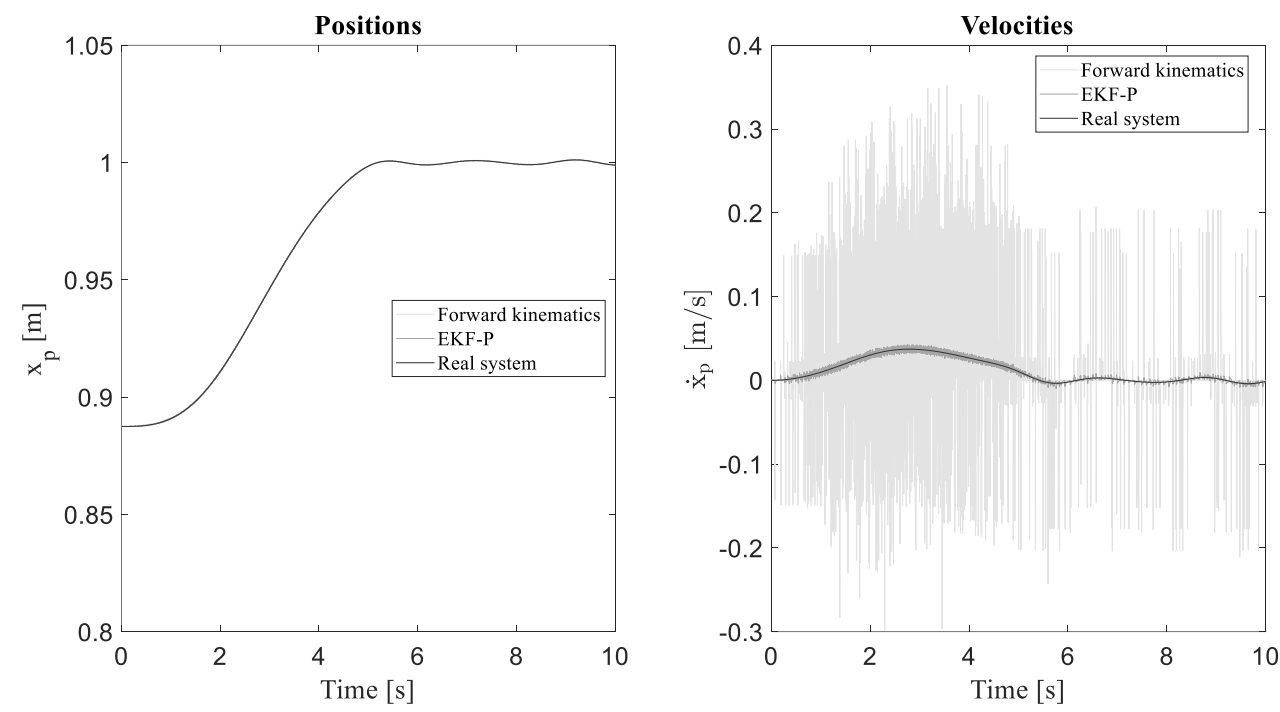

Figure 3. Comparison of actual and estimated $x_{p}$ and $\dot{x}_{p}$
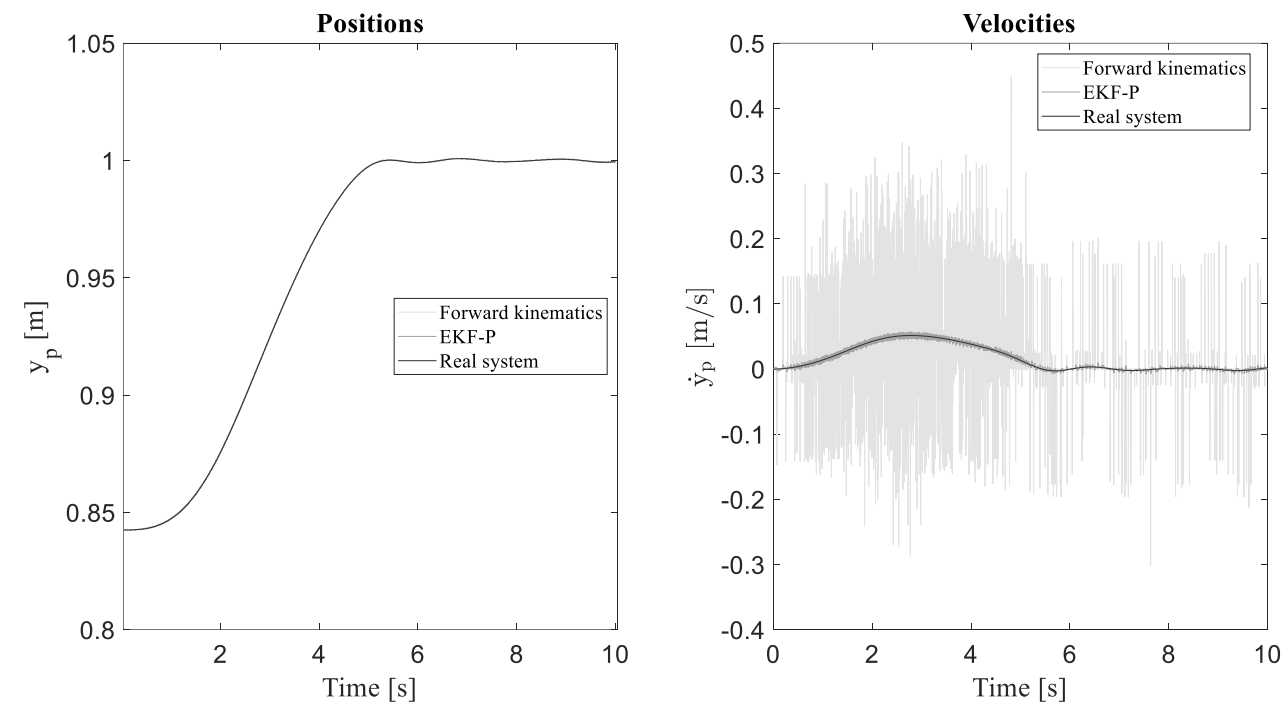

Figure 4. Comparison of actual and estimated $y_{p}$ and $\dot{y}_{p}$ 

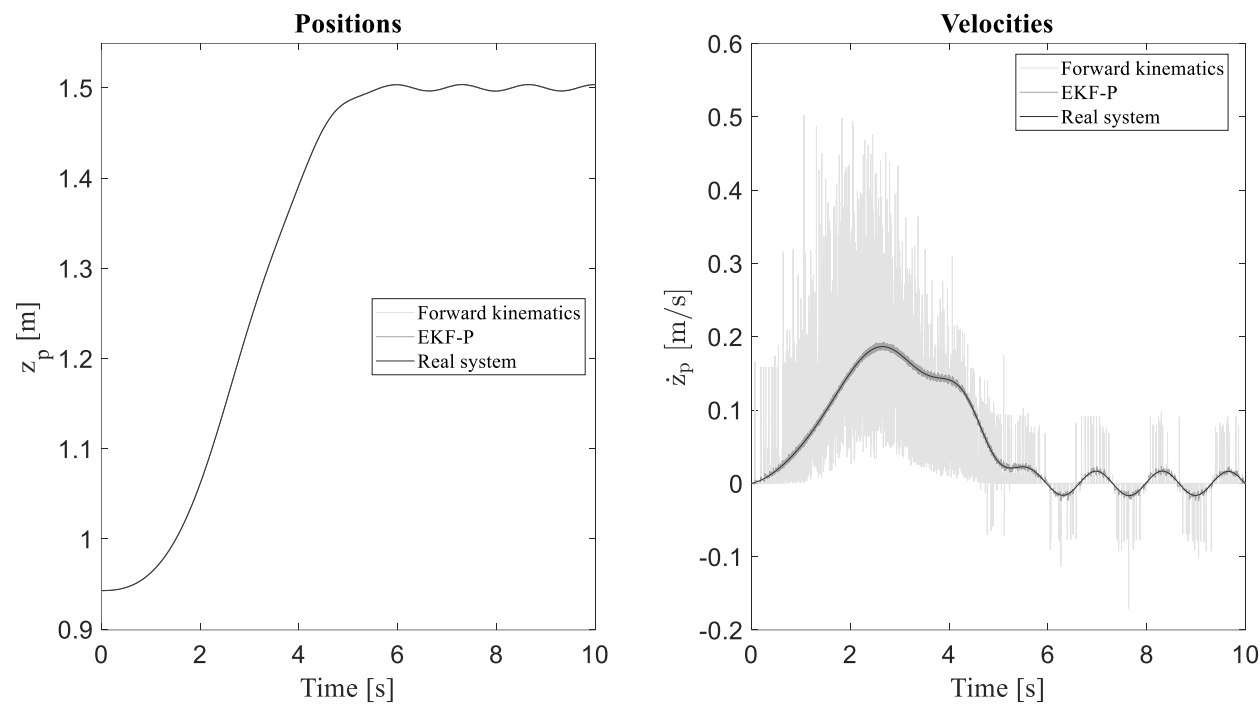

Figure 5. Comparison of actual and estimated $z_{p}$ and $\dot{z}_{p}$
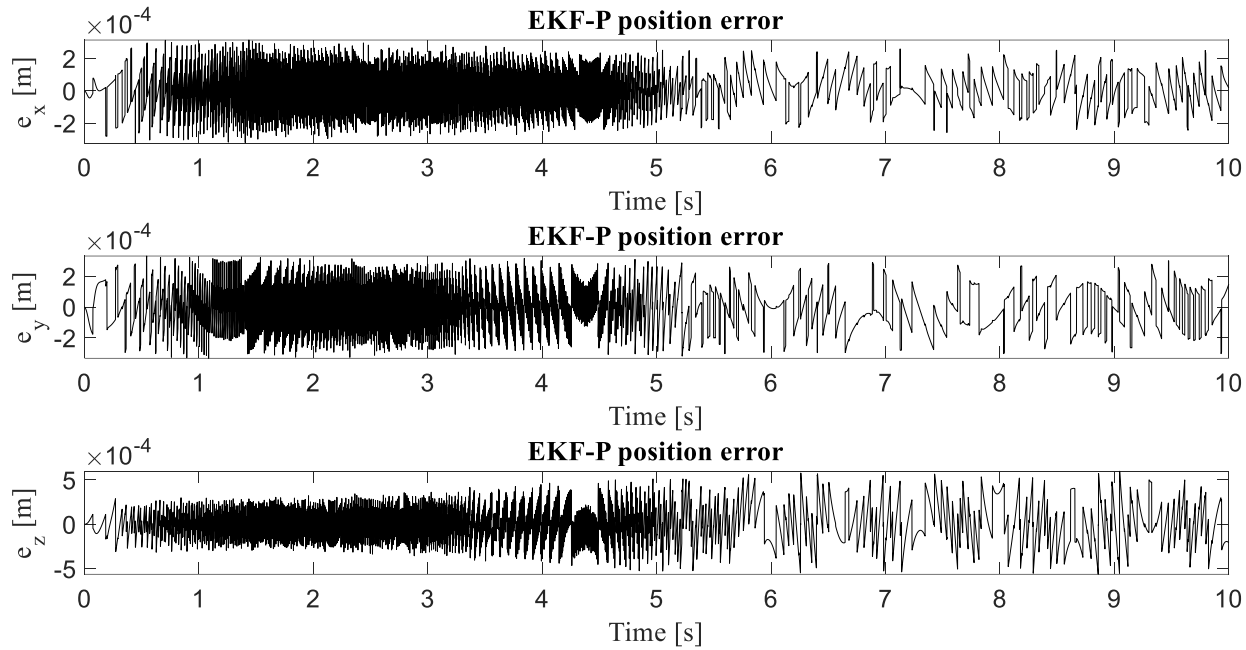

Figure 6. Time-history of position estimation errors of the EKF-P

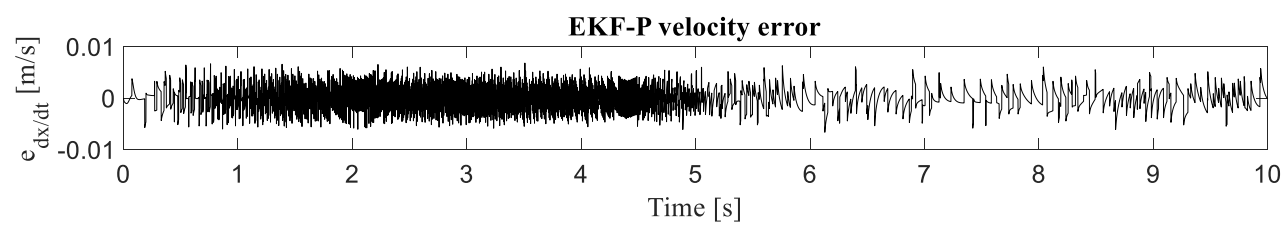

EKF-P velocity error

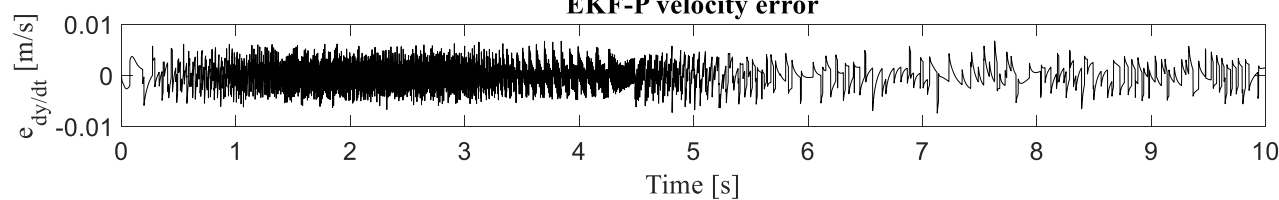

EKF-P velocity error

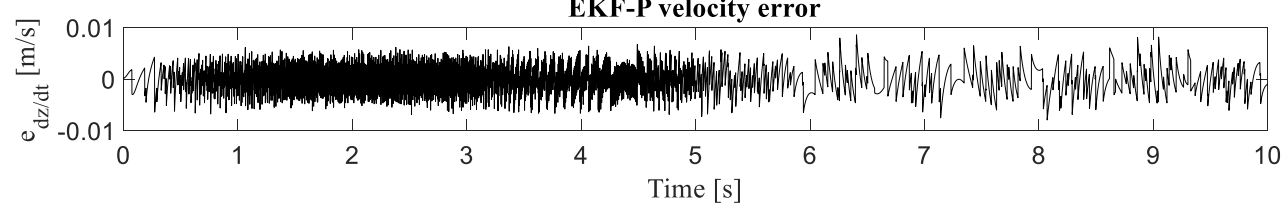

Figure 7. Time-history of velocity estimation errors of the EKF-P 


\subsection{State observer based on Udwadia-Kalaba formulation: EKF-UK}

The Udwadia-Kalaba formulation has been implemented as well, leading to the observer hereafter denoted as EKF-UK. The results of the simulation are compared with the actual values, and the estimation errors are plotted in Figures 8 and 9. The results are very similar with those provided by the EKF-P, and an effective speed noise rejection is, again, obtained.

Table 2 allows comparing the three different estimation approaches. While similar errors are obtained in term of position, the use of both the EKFs drastically reduces the speed RMS estimation error.
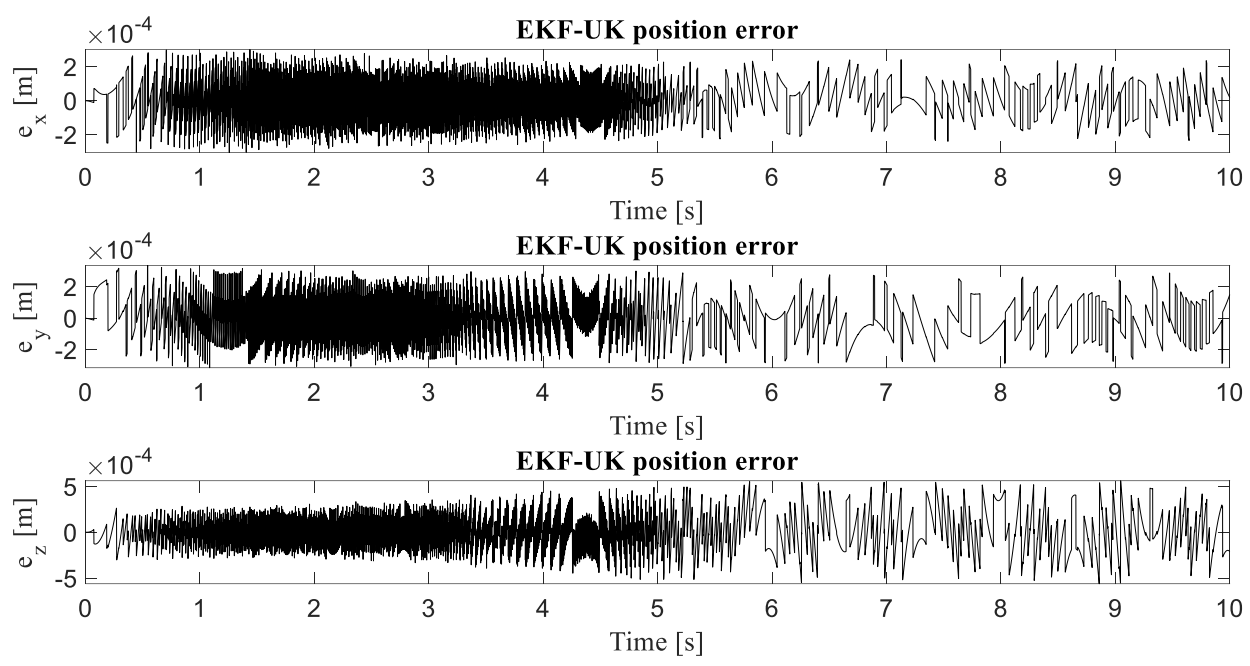

Figure 8. Time-history of position estimation errors of the EKF-UK

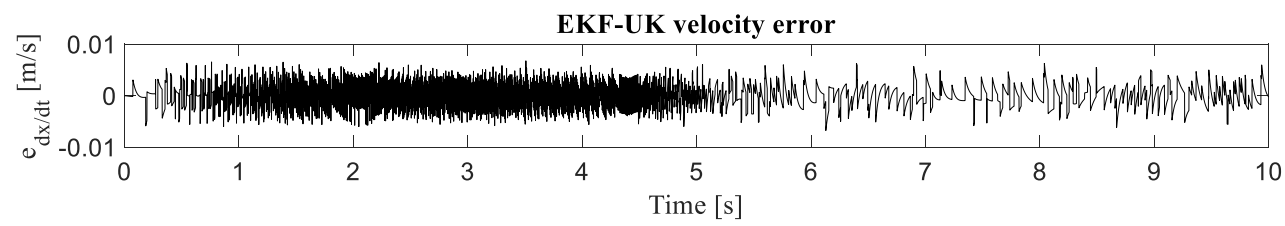

EKF-UK velocity error

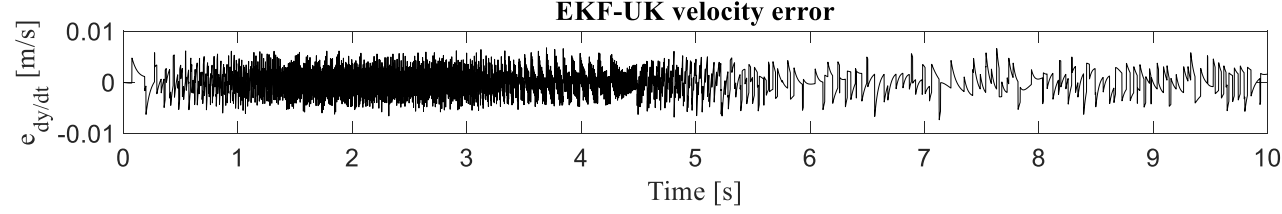

EKF-UK velocity error

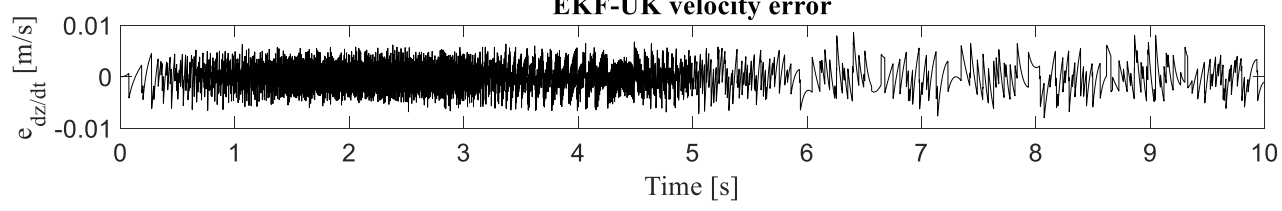

Figure 9. Time-history of velocity estimation errors of the EKF-UK

Table 2. RMS errors of position and velocity estimates against "real system"

\begin{tabular}{cccccccc}
\hline & EKF-P & EKF-UK & $\begin{array}{c}\text { Forward } \\
\text { kinematics }\end{array}$ & EKF-P & EKF-UK & $\begin{array}{c}\text { Forward } \\
\text { kinematics }\end{array}$ \\
\hline$e_{x}^{R M S}[\mathrm{~m}]$ & $1.13 \mathrm{e}-4$ & $1.08 \mathrm{e}-4$ & $1.20 \mathrm{e}-4$ & $e_{d x / d t}^{R M S}[\mathrm{~m} / \mathrm{s}]$ & $2.13 \mathrm{e}-3$ & $2.18 \mathrm{e}-3$ & 0.064 \\
$e_{y}^{R M S}[\mathrm{~m}]$ & $1.32 \mathrm{e}-4$ & $1.28 \mathrm{e}-4$ & $1.26 \mathrm{e}-4$ & $e_{d y / d t}^{R M S}[\mathrm{~m} / \mathrm{s}]$ & $2.24 \mathrm{e}-3$ & $2.29 \mathrm{e}-3$ & 0.059 \\
$e_{z}^{R M S}[\mathrm{~m}]$ & $2.04 \mathrm{e}-4$ & $1.99 \mathrm{e}-4$ & $2.43 \mathrm{e}-4$ & $e_{d z / d t}^{R M S}[\mathrm{~m} / \mathrm{s}]$ & $2.53 \mathrm{e}-3$ & $2.55 \mathrm{e}-3$ & 0.055 \\
\hline
\end{tabular}




\subsection{Estimation in the presence of model uncertainty}

A sensitivity analysis on the two EKFs has also been carried out by assuming random bounded perturbations of $\mathbf{M}$ (ranging in the interval $[0,+10 \%]$ ). Despite the relevant mismatch between the actual system model and the ones used in the state observer, a negligible increase of the estimation error is obtained, as shown in Table 3.

Table 3. RMS errors of position and velocity with model mismatch

\begin{tabular}{cccccc}
\hline & EKF-P & EKF-UK & & EKF-P & EKF-UK \\
\hline$e_{x}^{R M S}[\mathrm{~m}]$ & $1.13 \mathrm{e}-4$ & $1.08 \mathrm{e}-4$ & $e_{d x / d t}^{R M S}[\mathrm{~m} / \mathrm{s}]$ & $2.16 \mathrm{e}-3$ & $2.21 \mathrm{e}-3$ \\
$e_{y}^{R M S}[\mathrm{~m}]$ & $1.30 \mathrm{e}-4$ & $1.27 \mathrm{e}-4$ & $e_{d y / d t}^{R M S}[\mathrm{~m} / \mathrm{s}]$ & $2.23 \mathrm{e}-3$ & $2.28 \mathrm{e}-3$ \\
$e_{z}^{R M S}[\mathrm{~m}]$ & $2.04 \mathrm{e}-4$ & $1.99 \mathrm{e}-4$ & $e_{d z / d t}^{R M S}[\mathrm{~m} / \mathrm{s}]$ & $2.58 \mathrm{e}-3$ & $2.60 \mathrm{e}-3$ \\
\hline
\end{tabular}

\section{CONCLUSIONS}

This work discusses the synthesis of two formulations of EKFs for the state estimations in CableDriven Parallel Robot by means of some different multibody formulations. The approach is general and can be applied to several configurations Cable-Driven Parallel Robot. Among the two formulations presented to obtain ODEs from DAEs of the multibody model, the Udwadia-Kalaba formulation has shown some advantages due to a lower number of parameters to be tuned by the designer compared to the penalty formulation, that has led to a faster and simple tuning of the model adopted for the filter design. Overall, the performances of EKF-P and EKF-UK are comparable, and in both cases the computational effort wase small enough to allow for real time computation.

\section{ACKNOWLEDGMENTS}

The authors acknowledge the financial support by the Italian Ministry of University and Research through the research grant "SISTEMA - Dipartimenti di Eccellenza".

\section{REFERENCES}

[1] Boschetti, G., Minto, R. and Trevisani, A.: Improving a cable robot recovery strategy by actuator dynamics. Applied Sciences (Switzerland) (2020) 10 1-19

[2] Trevisani, A.: Planning of dynamically feasible trajectories for translational, planar, and underconstrained cable-driven robots. Journal of Systems Science and Complexity (2013) 26 695-717

[3] Idà, E., Briot, S. and Carricato, M.: Robust Trajectory Planning of Under-Actuated Cable-Driven Parallel Robot with 3 Cables. Springer Proceedings in Advanced Robotics (2021) 15 pp 65-72

[4] Albus, J., Bostelman, R. and Dagalakis, N.: The NIST robocrane. Journal of Robotic Systems (1993) $10709-24$

[5] Rosati, G., Zanotto, D. and Agrawal, S. K.: On the Design of Adaptive Cable-Driven Systems. Journal of Mechanisms and Robotics (2011) 3

[6] Ida, E., Marian, D. and Carricato, M.: A Deployable Cable-Driven Parallel Robot with Large Rotational Capabilities for Laser-Scanning Applications. IEEE Robotics and Automation Letters (2020) 5 4140-7

[7] Sun, C., Gao, H., Liu, Z., Xiang, S., Yu, H., Li, N. and Deng, Z.: Design and optimization of threedegree-of-freedom planar adaptive cable-driven parallel robots using the cable wrapping phenomenon. Mechanism and Machine Theory (2021) 166104475

[8] Lamaury, J. and Gouttefarde, M.: Control of a large redundantly actuated cable-suspended parallel robot. Proceedings - IEEE International Conference on Robotics and Automation (2013) 4659-64

[9] Jiang, Q. and Kumar, V.: The inverse kinematics of cooperative transport with multiple aerial robots. IEEE Transactions on Robotics (2013) 29 136-45

[10] Gagliardini, L., Caro, S., Gouttefarde, M. and Girin, A.: Discrete reconfiguration planning for Cable- 
Driven Parallel Robots. Mechanism and Machine Theory (2016) 100 313-37

[11] Belotti, R., Richiedei, D., Tamellin, I. and Trevisani, A.: Pole assignment for active vibration control of linear vibrating systems through Linear Matrix Inequalities. Applied Sciences (Switzerland) (2020) 10

[12] Richiedei, D. and Tamellin, I.: Active control of linear vibrating systems for antiresonance assignment with regional pole placement. Journal of Sound and Vibration (2021) 494115858

[13] Palomba, I., Richiedei, D. and Trevisani, A.: Kinematic state estimation for rigid-link multibody systems by means of nonlinear constraint equations. Multibody System Dynamics (2017) 40 1-22

[14] Pastorino, R., Richiedei, D., Cuadrado, J. and Trevisani, A.: State estimation using multibody models and non-linear Kalman filters. International Journal of Non-Linear Mechanics (2013) 53 83-90

[15] González, F. and Kövecses, J.: Use of penalty formulations in dynamic simulation and analysis of redundantly constrained multibody systems. Multibody System Dynamics (2013) 29 57-76

[16] Udwadia, F. E., Kalaba, R. E. and Phohomsiri, P.: Mechanical systems with nonideal constraints: Explicit equations without the use of generalized inverses. Journal of Applied Mechanics, Transactions ASME (2004) 71 615-21

[17] Yang, L., Xue, S. and Yao, W.: Application of Gauss principle of least constraint in multibody systems with redundant constraints. Proceedings of the Institution of Mechanical Engineers, Part K: Journal of Multi-body Dynamics (2020) 235 150-63

[18] Jalon, J. G. de. and Bayo, E.: Kinematic and Dynamic Simulation of Multibody Systems. SpringerVerlag, New York, NY (1994) ISBN:3-540-94096-0

[19] Riechel, A. T. and Ebert-Uphoff, I.: Force-Feasible Workspace analysis for underconstrained, pointmass cable robots. Proceedings - IEEE International Conference on Robotics and Automation (2004) vol 2004 pp 4956-62

[20] Falco, D. De, Pennestrì, E. and Vita, L.: The udwadia-kalaba formulation: A report on its numerical efficiency in multibody dynamics simulations and on its teaching effectiveness. Multibody Dynamics, ECCOMAS Thematic Conference (2005) 21-4

[21] Baumgarte, J.: Stabilization of constraints and integrals of motion in dynamical systems. Computer Methods in Applied Mechanics and Engineering (1972) 1 1-16

[22] Udwadia, F. E. and Phohomsiri, P.: Explicit equations of motion for constrained mechanical systems with singular mass matrices and applications to multi-body dynamics. Proceedings of the Royal Society A: Mathematical, Physical and Engineering Sciences (2006) 462 2097-117 